\title{
Predictive value of vascular endothelial growth factor-C expression for local recurrence of rectal carcinoma
}

\author{
EIJI NODA, KIYOSHI MAEDA, TORU INOUE, TAMAHIRO NISHIHARA, \\ YUKIO NISHIGUCHI, MASAICHI OHIRA and KOSEI HIRAKAWA \\ Department of Surgical Oncology, Osaka City University Graduate School \\ of Medicine, 1-5-7 Asahimachi Abeno-ku, Osaka 545-8585, Japan
}

Received January 4, 2007; Accepted February 20, 2007

\begin{abstract}
Vascular endothelial growth factor-C (VEGF-C) is considered to be a specific factor promoting lymphangiogenesis. There have been reports of a positive correlation between local recurrence of rectal carcinoma and lymphatic invasion by the tumor. The aim of this study was to determine the clinical significance of VEGF-C expression for identifying lymphangiogenesis as a predictor of the local recurrence of rectal carcinoma. One hundred surgical specimens of rectal carcinoma from patients with $(n=26)$ or without $(n=74)$ local recurrence were studied. VEGF-C protein expression was assessed immunohistochemically. The correlations between VEGF-C expression, various clinicopathologic factors, the microscopic lymphatic vessel density (MLVD), and lymphatic invasion were studied. The MLVD (determined by immunohistostaining for D2-40) was significantly higher in VEGF-C positive tumors than VEGF-C negative tumors. VEGF-C positivity was only correlated with lymphatic involvement. Moreover, multivariate analysis showed that VEGF-C protein expression was an independent risk factor for the local recurrence of rectal carcinoma, and patients with VEGF-C positive tumors had a significantly worse prognosis than those with VEGF-C negative tumors. Expression of VEGF-C may be a good predictor of the local recurrence of rectal carcinoma and may also be a useful prognostic indicator.
\end{abstract}

\section{Introduction}

VEGF-C was initially identified as a factor that stimulated tyrosine kinase receptor VEGFR-3, which was purified from PC-3 prostatic adenocarcinoma cells (1), VEGF-C is considered to be both a specific marker of lymphatic endothelial

Correspondence to: Dr Eiji Noda, Department of Surgical Oncology, Osaka City University, Graduate School of Medicine, 1-5-7 Asahimachi Abeno-ku, Osaka 545-8585, Japan

E-mail: nodatch@med.osaka-cu.ac.jp

Key words: vascular endothelial growth factor-C, lymphatic invasion, local recurrence, rectal carcinoma, predictive factor, prognosis, immunohistochemistry cells and a specific factor that promotes lymphangiogenesis. Expression of VEGF-C has been correlated with lymph node metastasis in patients who have breast (2), colorectal (3), gastric (4), thyroid (5), lung (6) and prostate (7) cancer.

Local recurrence is a major problem after the surgical treatment of rectal carcinoma because it occurs frequently and is invariably associated with a poor prognosis. Michelassi et al reported a positive relationship between the local recurrence of rectal carcinoma and tumor invasion of lymphatic channels (8). Therefore, it has been suggested that VEGF-C expression may be a predictor of the local recurrence of rectal carcinoma, and may also be a useful prognostic indicator for this carcinoma.

On the other hand, it is difficult to identify lymphatic vessels and lymphatic involvement by examination of sections after conventional hematoxylin and eosin staining. Over the last few years, an increasing number of factors have been proposed as specific markers for the developing lymphatic endothelium. These factors associated with lymphangiogenesis include VEGF-C, VEGF-D, VEGFR-2, VEGFR-3, LYVE-1, Prox-1, podoplanin and 5'-nucleotidase. A new monoclonal antibody, D2-40 (IgG1), that targets an Mr 40,000 O-linked sialoglycoprotein was previously purified from ascites (9). D2-40 selectively reacts with the endothelium of lymphatics. Kahn et al reported that D2-40 clearly demarcated tumor emboli in lymphatics when examining paraffin-embedded sections of primary tumors (10). Therefore, this monoclonal antibody can be used to increase the accuracy of detecting lymphatic invasion.

The aim of this study was to investigate the clinical significance of VEGF-C expression as a predictor of the local recurrence and prognosis of rectal carcinoma.

\section{Materials and methods}

We studied 100 patients with rectal carcinoma who underwent anterior resection at the Department of Surgical Oncology (First Department of Surgery) of Osaka City University Graduate School of Medicine (Osaka, Japan). Table I shows their characteristics. The age of the patients ranged from 39 to 85 years (mean 63.5 years), and there were 64 men and 36 women. None of the patients received chemotherapy or radiation therapy before surgery. All of them underwent curative resection and were followed for at least 5 years after 
Table I. Clinical characteristics of the patients and correlation between VEGF-C expression and various clinicopathlogic factors. ${ }^{\mathrm{a}}$

\section{VEGF-C expression}

(+) (-) P-value

\section{Gender}

Male

Female

Age

$$
\text { Median (range) }
$$

$\mathrm{Ra}$

$\mathrm{Rb}$

Tumor diameter (mm)

Median (range)

$50.0(13-115)$

Moderate

Poor

Mucinous

Depth of invasion

$\mathrm{T} 2$

$\mathrm{T} 3, \mathrm{~T} 4$

Lymphatic involvement

Positive

Negative

Venous involvement

Positive

Negative

Lymph node metastasis

Positive

Negative

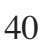

55

64

6

41

2

3

18

82

56

44

14

86

63
21

15

$64.0(51-85)$

11

8

17

$47.5(13-72)$

$50.0(16-115)$

NS

NS

22

1

1

6

30

30

6

26

38

10

54

32

14

23

NS

${ }^{\mathrm{a} E x c e p t}$ for with age and tumor diameter, data show the number of patients.

surgery, with routine diagnostic imaging (computed tomography, ultrasonography or magnetic resonance imaging) two or three times per year. All patients were informed of the investigational nature of this study and provided written informed consent before registration.

Immunohistochemistry. Immunohistochemical studies were performed on formalin-fixed, paraffin-embedded sections using anti-VEGF-C and D2-40 antibodies. Sections were cut at a thickness of $4 \mu \mathrm{m}$, dried for $4 \mathrm{~h}$ at $58^{\circ} \mathrm{C}$, and then dewaxed in xylene and dehydrated through an ethanol series. Endogenous peroxidase was blocked by incubation with $0.3 \%$ $\mathrm{H}_{2} \mathrm{O}_{2}$ in methanol for $30 \mathrm{~min}$ at room temperature. Then the sections were microwaved 3 times (at $500 \mathrm{~W}$ for $5 \mathrm{~min}$ each) in $10 \mathrm{mM}$ sodium citrate (pH 6.0). After washing with phosphate-buffered saline (PBS), sections were incubated with $10 \%$ normal goat or rabbit serum for $30 \mathrm{~min}$ to reduce non-specific staining. Then a polyclonal rabbit anti-VEGF-C antibody (Zymed Laboratories, South San Francisco, CA) was applied to the sections at a dilution of 1:100 and incubation was conducted in a moist chamber overnight at $4{ }^{\circ} \mathrm{C}$. The mouse monoclonal antibody D2-40, which had been already diluted by the manufacturer (Nichirei, Tokyo, Japan), was applied to the sections and incubation was conducted in a moist chamber overnight at $4^{\circ} \mathrm{C}$. After washing the specimens with PBS, the slides were incubated with biotinylated goat anti-rabbit antibody or biotinylated rabbit anti-mouse antibody for $30 \mathrm{~min}$ at room temperature. After three washes in PBS, 
a
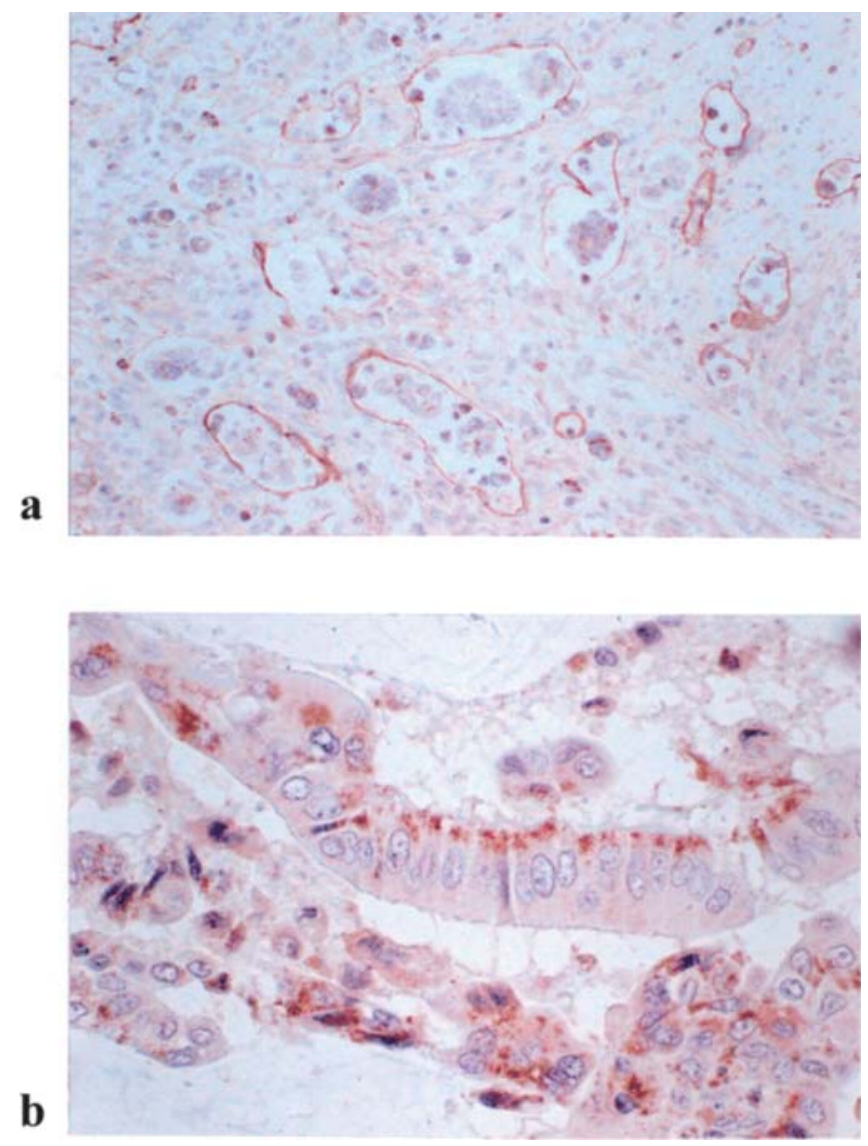

Figure 1. (a) Identifying lymphatic invasion by immunostaining with D2-40. There is strong staining of the lymphatic endothelium, and tumor cells can be identified inside the lymphatics (original magnification x200). (b) VEGF-C expression in rectal carcinoma. VEGF-C immunoreactivity is present in the cytoplasm of tumor cells (original magnification x200).

sections were incubated with the streptavidin-peroxidase reagent for $5 \mathrm{~min}$ at room temperature. Finally, the sections were incubated for $5 \mathrm{~min}$ in PBS containing diaminobenzidine and $1 \%$ hydrogen peroxide (Histofine SAB-PO kit, Nichirei), followed by counterstaining with Mayer's hematoxylin. As the negative control, incubation with the primary antibody was omitted.

Evaluation of VEGF-C immunoreactivity. Slides were examined by two investigators who were without knowledge of the corresponding clinicopathologic data. Any discrepant scores were re-evaluated jointly on a second occasion, and agreement was reached.

Microscopic lymphatic vessel density (MLVD) and identification of lymphatic invasion. Sections stained by D2-40 were viewed at a $\times 40$ magnification to identify the regions of highest vascularity within the tumor. After three areas were identified, vessel counting was performed at a x100 magnification and the average count of three fields was calculated. Lymphatic invasion was identified with immunostaining by D2-40 (Fig. 1a).

Statistical analysis. Statistical analysis was performed using JMP software version 5.1.2 (SAS Institute, Inc., Cary, NC, USA). The relationship between VEGF-C expression and

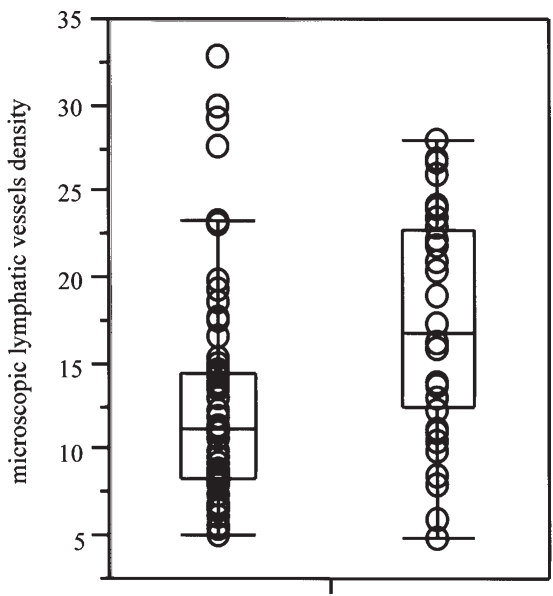

$(-)$

$(+)$

Expression of VEGF-C

Figure 2. Correlation between microscopic lymphatic vessel density (MLVD) and VEGF-C expression. The ends of the box are the 25 and $75 \%$ quantiles and the line across the middle of the box identifies the median sample value (box and whisker plots). MLVD of the VEGF-C positive tumors was significantly higher than that of the VEGF-C negative tumors $(\mathrm{P}<0.001)$.

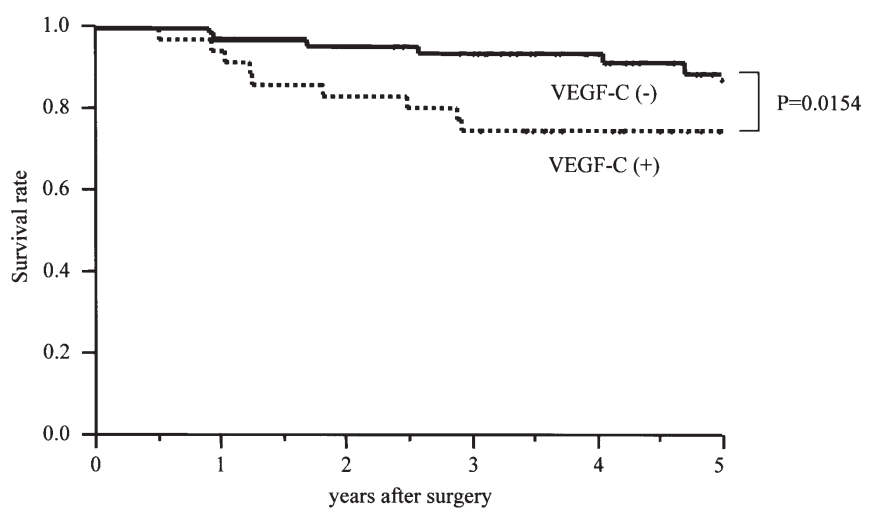

Figure 3. Survival rate after curative resection. Overall survival was significantly worse in the patients with VEGF-C positive tumors.

the microscopic lymphatic vessel density was evaluated by Wilcoxon's test. For univariate and multivariate analysis of the correlation between local recurrence of rectal carcinoma and various clinicopathologic factors or VEGF-C expression, logistic regression was used. Survival curves were calculated by the Kaplan-Meier method and differences in survival were analyzed by the log-rank test.

\section{Results}

VEGF-C expression was mainly localized to the cytoplasm of the cancer cells (Fig. 1b). Among the 100 tumors examined, $36(36.0 \%)$ showed VEGF-C expression. Table I shows the correlations between VEGF-C expression and various clinicopathologic factors. Among them, VEGF-C protein expression in the primary tumor only had a statistically significant relationship with lymphatic involvement $(\mathrm{P}<0.001)$.

Based on the relation between MLVD and VEGF-C expression, the median MLVD of VEGF-C positive tumors was 16.9, 
Table II. Correlation between local recurrence and various clinicopathologic factors, including microscopic lymphatic vessel density and VEGF-C expression.

\begin{tabular}{lccccccc}
\hline & \multicolumn{3}{c}{ Univariate analysis } & & \multicolumn{3}{c}{ Multivariate analysis } \\
\cline { 2 - 3 } & Odds ratio & $95 \% \mathrm{CI}$ & P-value & & Odds ratio & $95 \%$ CI & P-value \\
\hline VEGF-C expression & 4.320 & $1.71-11.4$ & 0.0023 & & 5.319 & $1.61-20.3$ & 0.0087 \\
Lymphatic involvement & 4.680 & $1.70-15.2$ & 0.0050 & & 2.158 & $0.62-8.26$ & 0.2355 \\
Venous involvement & 7.306 & $2.24-26.5$ & 0.0013 & 12.133 & $2.94-59.8$ & 0.0010 \\
Lymph node metastasis & 2.587 & $1.04-6.56$ & 0.0417 & & 2.764 & $0.95-8.46$ & 0.0660 \\
\hline
\end{tabular}

which was significantly higher than that for VEGF-C negative tumors $(\mathrm{P}<0.001)$ (Fig. 2).

Assessment of the prognosis showed that the overall survival was significantly worse in patients with VEGF-C positive tumors (Fig. 3).

Among 26 patients who had local recurrence, 17 died of progressive disease. Table II shows the correlations between local recurrence and various clinicopathologic factors, including MLVD and VEGF-C expression. On univariate analysis, local recurrence had a significant relationship with venous involvement, lymph node metastasis, lymphatic involvement and VEGF-C expression. However, multivariate analysis indicated that only VEGF-C protein expression and venous involvement were independent risk factors for the local recurrence of rectal carcinoma.

\section{Discussion}

Local recurrence is a major problem in patients undergoing the surgical treatment of rectal carcinoma because it is frequent and always associated with a poor prognosis $(11,12)$. Therefore, prediction of local recurrence could be extremely important for the management of rectal carcinoma and better local control could improve the quality of life after surgery.

In this study, expression of VEGF-C protein was not only shown to be a prognostic indicator, but was also useful for predicting the local recurrence of rectal carcinoma. Furthermore, this report is the first to indicate a clinical utility of VEGF-C expression of rectal carcinoma.

Some earlier studies have suggested a significant relationship between local recurrence and lymphatic invasion or lymph node metastasis in patients with rectal carcinoma. Michelassi et al (8) studied 853 colorectal cancer patients and reported that the local recurrence rate significantly correlated with microscopic lymphatic or capillary invasion. In the present study, we also found that local recurrence was significantly more frequent in patients with lymphatic invasion.

VEGF-C is a ligand for VEGFR-3 and is thought to be a specific inducer of lymphangiogenesis (1). A study using transgenic mice has shown that the VEGF-C/VEGFR-3 signaling pathway is essential for the growth of lymphatic vessels (13). Kawakami et al (14) reported that VEGF-C m-RNA and protein were highly expressed in patients with colorectal cancer who had lymph node metastasis and lymphatic invasion. Our findings also indicated that VEGF-C expression was significantly correlated with lymphatic invasion.
The monoclonal antibody D2-40 reacts selectively with an O-linked sialoglycoprotein that is specific for lymphatic vessels. Kahn et al $(10,15)$ studied 50 breast cancer patients and reported that D2-40 was superior for detecting lymphatic invasion in comparison with hematoxylin and eosin staining, being more specific and more accurate. Therefore, we used D2-40 for lymphatic identification in the present study. As a result, we found that VEGF-C-positive tumors had a high MLVD. Jia et al (16) previously reported a positive correlation between lymphatic density and VEGF-C expression in 57 patients with colorectal cancer. Rubbia-Brandt et al (17) also found similar results in 39 patients with pancreatic endocrine tumors. Thus, it has been suggested that VEGF-C induces lymphangiogenesis at the tumor border allowing cancer cells to easily invade the proliferating lymphatic vessels.

With regard to the influence of VEGF-C on prognosis, several previous reports have indicated that VEGF-C expression is an independent and significant prognostic factor for patients with colorectal carcinoma $(18,19)$, and the same result was obtained in our study. We also found that VEGF-C expression strongly correlated with local recurrence, and multivariate analysis indicated that VEGF-C expression was an independent predictor of local recurrence.

Various regimens of adjuvant chemotherapy have been administered to patients with advanced colorectal carcinoma in an attempt to prevent recurrence after resection. It is possible that analysis of VEGF-C expression in resected tumor specimens may provide additional guidance in identifying the patients who most need adjuvant therapy.

Recently, it was reported that preoperative chemoradiotherapy (CRT) and total mesorectal excision (TME) (20-24) could significantly reduce the local recurrence rate and increase overall survival. Patients who need neoadjuvant therapy and operative methods such as TME should be selected using indicators of the risk of recurrence. Maeda et al (25) reported that VEGF-C staining of endoscopic biopsy specimens was useful for preoperative selection of treatment in patients with $\mathrm{T} 1$ colorectal carcinoma. Therefore, investigation of VEGF-C expression in preoperative biopsy specimens may be useful to select high-risk patients for local recurrence who need preoperative CRT and TME.

In conclusion, this retrospective study demonstrated that VEGF-C expression is an important predictor of the local recurrence of rectal carcinoma. If these findings are confirmed by a larger study, it might be possible to identify patients with 
a high risk of local recurrence and use this information to plan their therapy.

\section{References}

1. Joukov V, Pajusola K, Kaipainen A, Chilov D, Lahtinen I, Kukk E, Saksela O, Kalkkinen N and Alitalo K: A novel vascular endothelial growth factor, VEGF-C, is a ligand for the Flt4 (VEGFR-3) and KDR (VEGFR-2) receptor tyrosine kinases. EMBO J 15: 1751, 1996

2. Kurebayashi J, Otsuki T, Kunisue H, Mikami Y, Tanaka K, Yamamoto $\mathrm{S}$ and Sonoo $\mathrm{H}$ : Expression of vascular endothelial growth factor (VEGF) family members in breast cancer. Jpn J Cancer Res 90: 977-981, 1999.

3. Akagi K, Ikeda Y, Miyazaki M, Abe T, Kinoshita J, Maehara Y and Sugimachi K: Vascular endothelial growth factor-C (VEGF-C) expression in human colorectal cancer tissues. Br J Cancer 83: 887-891, 2000.

4. Yonemura Y, Endo Y, Fujita H, Fushida S, Ninomiya I, Bandou E, Taniguchi K, Miwa K, Ohoyama S, Sugiyama K and Sasaki T: Role of vascular endothelial growth factor $C$ expression in the development of lymph node metastasis in gastric cancer. Clin Cancer Res 5: 1823-1829, 1999.

5. Bunone G, Vigneri P, Mariani L, Buto S, Collini P, Pilotti S, Pierotti MA and Bongarzone I: Expression of angiogenesis stimulators and inhibitors in human thyroid tumors and correlation with clinical pathological features. Am J Pathol 155: 1967-1976, 1999

6. Kajita T, Ohta Y, Kimura K, Tamura M, Tanaka Y, Tsunezuka Y, Oda M, Sasaki T and Watanabe G: The expression of vascular endothelial growth factor $\mathrm{C}$ and its receptors in non-small cell lung cancer. Br J Cancer 85: 255-260, 2001.

7. Tsurusaki T, Kanda S, Sakai H, Kanetake H, Saito Y, Alitalo K and Koji T: Vascular endothelial growth factor-C expression in human prostatic carcinoma and its relationship to lymph node metastasis. Br J Cancer 80: 309-313, 1999.

8. Michelassi F, Vannucci L, Ayala JJ, Chappel R, Goldberg R and Block GE: Local recurrence after curative resection of colorectal adenocarcinoma. Surgery 108: 783-792, 1990.

9. Marks A, Sutherland DR, Bailey D, Iglesias J, Law J, Lei M, Yeger H, Banerjee D and Baumal R: Characterization and distribution of an oncofetal antigen (M2A antigen) expressed on testicular germ cell tumours. Br J Cancer 80: 569-578, 1999.

10. Kahn HJ and Marks A: A new monoclonal antibody, D2-40, for detection of lymphatic invasion in primary tumors. Lab Invest 82: 1255-1257, 2002.

11. Secco GB, Fardelli R, Campora E, Rovida S and Bertoglio S: Factors influencing local recurrence after curative surgery for rectal cancer. Oncology 46: 10-13, 1989.

12. Rinnert-Gongora S and Tartter PI: Multivariate analysis of recurrence after anterior resection for colorectal carcinoma. Am J Surg 157: 573-576, 1989.
13. Veikkola T, Jussila L, Makinen T, Karpanen T, Jeltsch M, Petrova TV, Kubo H, Thurston G, McDonald DM, Achen MG, Stacker SA and Alitalo K: Signalling via vascular endothelial growth factor receptor-3 is sufficient for lymphangiogenesis in transgenic mice. EMBO J 20: 1223-1231, 2001.

14. Kawakami M, Furuhata T, Kimura Y, Yamaguchi K, Hata F, Sasaki K and Hirata K: Quantification of vascular endothelial growth factor-C and its receptor-3 messenger RNA with realtime quantitative polymerase chain reaction as a predictor of lymph node metastasis in human colorectal cancer. Surgery 133: 300-308, 2003.

15. Kahn HJ, Bailey D and Marks A: Monoclonal antibody D2-40, a new marker of lymphatic endothelium, reacts with Kaposi's sarcoma and a subset of angiosarcomas. Mod Pathol 15: 434-440, 2002.

16. Jia YT, Li ZX, He YT, Liang W, Yang HC and Ma HJ: Expression of vascular endothelial growth factor-C and the relationship between lymphangiogenesis and lymphatic metastasis in colorectal cancer. World J Gastroenterol 10: 3261-3263, 2004.

17. Rubbia-Brandt L, Terris B, Giostra E, Dousset B, Morel P and Pepper MS: Lymphatic vessel density and vascular endothelial growth factor-C expression correlate with malignant behavior in human pancreatic endocrine tumors. Clin Cancer Res 10: 6919-6928, 2004

18. Furudoi A, Tanaka S, Haruma K, Kitadai Y, Yoshihara M, Chayama K and Shimamoto F: Clinical significance of vascular endothelial growth factor $\mathrm{C}$ expression and angiogenesis at the deepest invasive site of advanced colorectal carcinoma. Oncology 62: $157-166,2002$.

19. Onogawa S, Kitadai Y, Tanaka S, Kuwai T, Kimura S and Chayama K: Expression of VEGF-C and VEGF-D at the invasive edge correlates with lymph node metastasis and prognosis of patients with colorectal carcinoma. Cancer Sci 95: 32-39, 2004.

20. Swedish Rectal Cancer Trial: Improved survival with preoperative radiotherapy in resectable rectal cancer. N Engl J Med 336: 980-987, 1997.

21. Camma C, Giunta M, Fiorica F, Pagliaro L, Craxi A and Cottone M: Preoperative radiotherapy for resectable rectal cancer: a meta-analysis. JAMA 284: 1008-1015, 2000.

22. Colorectal Cancer Collaborative Group: Adjuvant radiotherapy for rectal cancer: a systematic over-view of 8,507 patients from 22 randomised trials. Lancet 358: 1291-1304, 2001.

23. MacFarlane JK, Ryall RD and Heald RJ: Mesorectal excision for rectal cancer. Lancet 341: 457-460, 1993.

24. Enker WE, Thaler HT, Cranor ML and Polyak T: Total mesorectal excision in the operative treatment of carcinoma of the rectum. $\mathbf{J}$ Am Coll Surg 181: 335-346, 1995.

25. Maeda K, Yashiro M, Nishihara T, Nishiguchi Y, Sawai M, Uchima K, Onoda N, Ohira M, Ishikawa T and Hirakawa K: Correlation between vascular endothelial growth factor $\mathrm{C}$ expression and lymph node metastasis in T1 carcinoma of the colon and rectum. Surg Today 33: 736-739, 2003. 